\title{
Terapia asistida con vacío y estoma flotante (floating stoma). Una nueva forma de tratar un absceso periostomal
}

\author{
Arturo Vergara ${ }^{1}$, Manuel Cadena ${ }^{1}$, Luis Felipe Cabrera ${ }^{1}$ y Mauricio Pedraza ${ }^{2}$
}

\section{Vacuum assisted therapy and floating stoma. S new way to treat a periostomal abscess}

Introduction: Floating stomata are defined as a stoma that is not attached to the skin of the abdominal wall, this technique is described in surgery for entero-atmospheric fistulas and damage control. Clinical Case: We present a case report of the management of paraostomal abscess in a patient with a history of laparotomy due to acute abdomen, it was decided to use negative pressure therapy associated with a floating stoma, demonstrating its usefulness in this complex type of patient.

Key words: floating stoma; Bogota bag; damage control surgery; open abdomen; relaparotomies.

\section{Resumen}

Introducción: Los estomas flotantes se definen como un estoma que no está fijado a la piel de la pared abdominal, esta técnica se ha descrito en cirugía para fístulas entero-atmosféricas y de control de daños. Caso Clínico: Presentamos un reporte de caso de manejo de absceso paraostomal en paciente con antecedente de laparotomía por abdomen agudo, por lo que se decide uso de terapia de presión negativa asociada a estoma flotante, demostrando su utilidad en este tipo complejo de pacientes.

Palabras clave: estoma flotante; bolsa de Bogotá; cirugía de control de daños; abdomen abierto y relaparotomía.

\section{Introducción}

Las ostomías digestivas son comunicaciones artificiales de un órgano hueco intraperitoneal con la pared abdominal, mediante una intervención quirúrgica. Su finalidad es suplir las vías naturales de excreción o alimentación cuando éstas se interrumpen por cualquier causa clínica ${ }^{1,2}$.

Las ostomías digestivas reciben distintos nombres según la porción del tracto digestivo abocada, entre las que se encuentran: la gastrostomía, la yeyunostomía, ileostomía y colostomía. Según su función las dos primeras son conocidas como ostomías de alimentación y las segundas como ostomías de eliminación ${ }^{3,4}$.

En el presente trabajo, nos permitimos presentar un caso de absceso paraostomal manejado con terapia asistida con vacío y un estoma flotante, con una revisión crítica de la literatura, el cual queremos dar a conocer como una forma para manejar cavidades paraostomales complicadas, drenando el absceso, corrigiendo el espacio muerto y la retracción, sin necesidad de anestesia general o salas de cirugía, evitando los riesgos perioperatorios, la prolongación del tratamiento médico, el agotamiento y la frustración del equipo quirúrgico ${ }^{1-3}$.

\section{Caso Clínico}

Se trata de una mujer de 58 años con obesidad mórbida (índice de masa corporal (IMC) de $36 \mathrm{~kg}$ / $\mathrm{m}^{2}$ ), llevada de forma extrainstitucional a laparotomía exploratoria encontrando una diverticulitis aguda Hinchey tipo III del colon sigmoide, por lo cual realizan sigmoidectomía con colostomía tipo Hartmann. Ingresa remitida a nuestra institución al sexto día posoperatorio, con dehiscencia de la herida
${ }^{1}$ Fundación Santa Fe de Bogotá

¿Universidad El Bosque. Bogotá, Colombia.

Recibido 2020-03-07 y aceptado 2020-11-03

Correspondencia a: Dr. Mauricio Pedraza mpedraza93@gmail.com 


\section{CASOS CLÍNICOS}

Figura 1. Absceso periostomal y retracción ostomía. quirúrgica y múltiples abscesos intraabdominales (infección del sitio operatorio (ISO) tipo III, órgano/ espacio). Además, presenta absceso periostomal con retracción de la ostomía y dehiscencia de la sutura mucoepitelial.

Es llevada a salas de cirugía donde se realiza lavado quirúrgico con drenaje de colecciones, toma de muestra de líquido peritoneal para estudio microbiológico y se instala sistema $\mathrm{VAC}^{\circledR}$ (KCI Licensing, Inc. An Acelity Company) en herida de laparotomía, y se inicia antibioticoterapia empírica.

Secundario a las colecciones periostomales se formaron bolsillos amplios en $360^{\circ} \mathrm{y}$ de profundidad hasta la aponeurosis, con retracción de colostomía de forma secundaria (Figura 1). Por esto se decide realizar una neopared con Viaflex ${ }^{\circledR}$ (Baxter Healthcare Corporation para fijar la ostomía, evertiendo sobre la nueva pared su producción, limitando el drenaje de materia fecal a los bolsillos periostomales, lo cual se denomina "floating stoma" (Figura 2).

\section{Técnica quirúrgica}

El "floating stoma" se realizó de la siguiente forma: con material de Viaflex ${ }^{\circledR}$ (Baxter Healthcare Corporation) mayor al defecto de la pared, se realiza orificio central menor al diámetro de la ostomía, ya que este se aumenta al realizar la sutura. Esta se realizó primero con seda 3-0 y, posteriormente, con polipropileno 3-0, encontrando mejor resultado de resistencia con este último. Se realiza enterorrafia en corona con sutura continua evertiendo los bordes de la ostomía con puntos de Brooke. Se ubica esponja negra Granufoam del sistema VAC (KCI Licensing, Inc. An Acelity Company) en orificio periostomal completando el espacio de tejido perdido y se extrae en sentido al declive de la pared abdominal como se muestra en la Figura 3; se aplica lámina adhesiva del sistema VAC (KCI Licensing, Inc. An Acelity Company), creando un plano separado el cual sirve para hacer el vacío necesario. Se realiza un orificio en el adhesivo sobre la ostomía del tamaño necesario para poder retraerla alrededor. Se coloca sobre esta

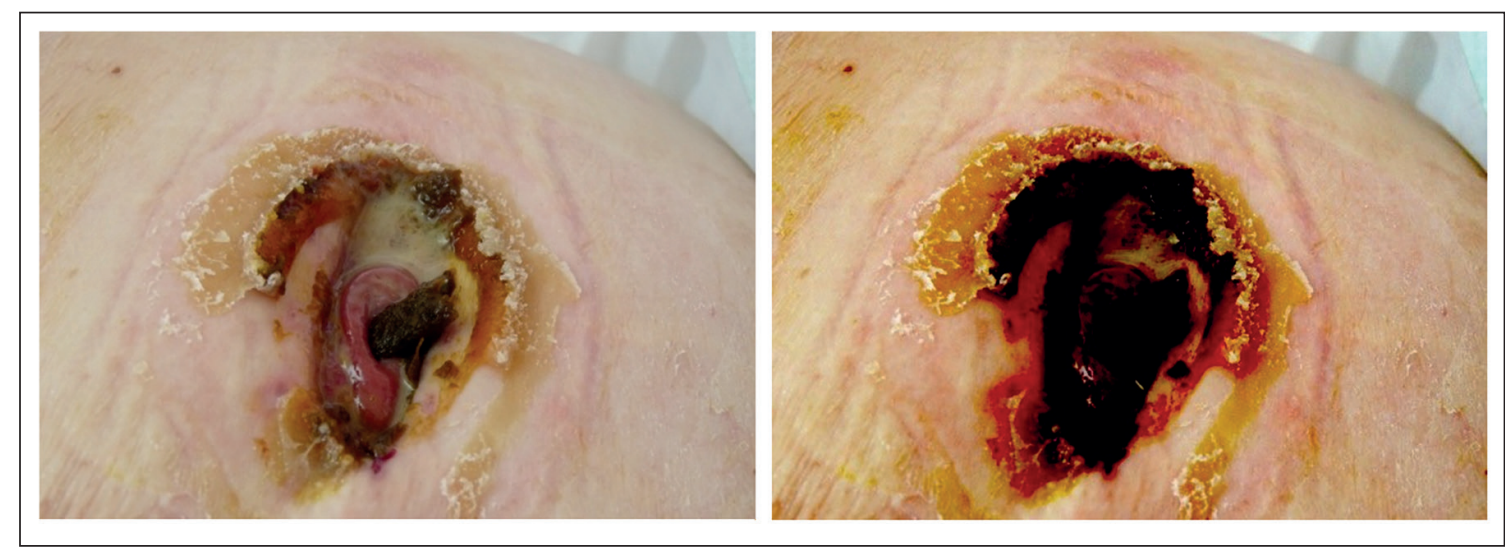

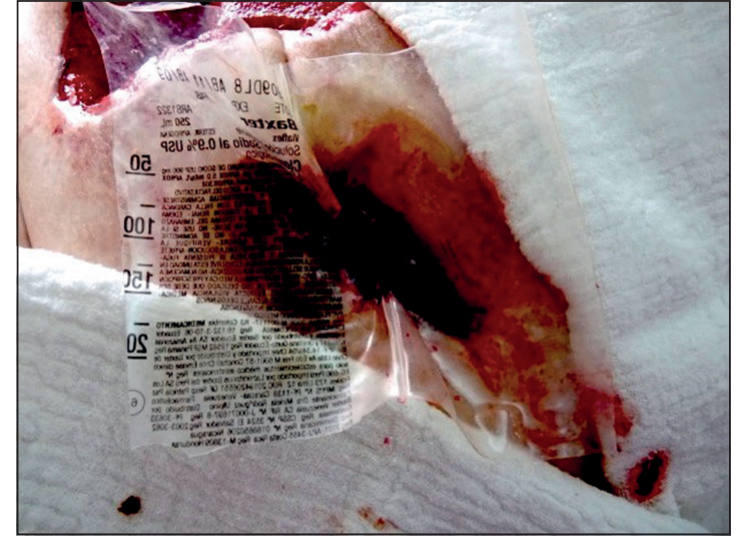

Figura 2. Estoma flotante. Neopared separando producción fecal y espacio periostomal.

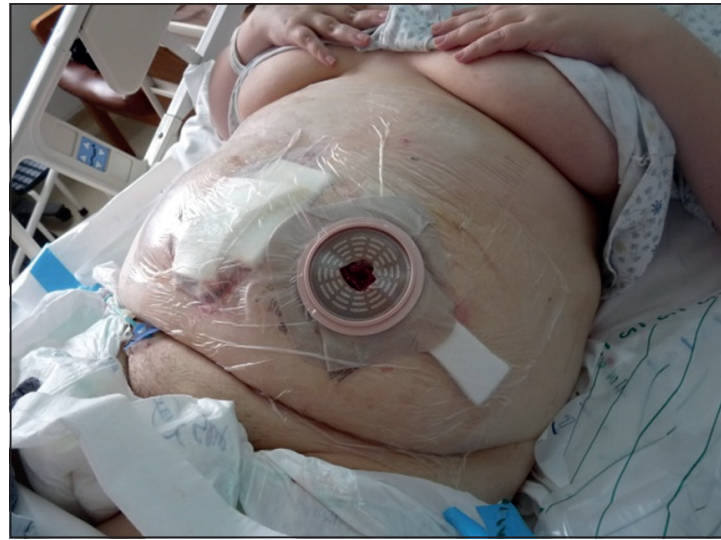

Figura 3. Sistema VAC colocado por planos. 
Stomahesive ${ }^{\circledR}$ pasta (Convatec Inc.) en $360^{\circ}$, con su respectiva barrera y bolsa de colostomía.

En nuestro paciente, el desafío radicó en la cercanía entre la herida de laparotomía y el lugar de exteriorización de la colostomía. Se realizó separación por planos mediante el adhesivo de las heridas respectivas y se colocaron dos sistemas de VAC (KCI Licensing, Inc. An Acelity Company) aislados, lo cual nos permitió el cierre de la lesión periostomal en 6 días con control infeccioso total y resolución de los abscesos, cierre del espacio muerto subcutáneo en su totalidad con corrección de la retracción y posterior remodelación de la colostomía, la cual se puede realizar en la cama del paciente. Se realizó cierre de la laparostomía en salas de cirugía, a los 20 días de iniciada la terapia con el sistema VAC (KCI Licensing, Inc. An Acelity Company) (Figura 4). Adicionalmente se trató una colección residual en pelvis supravesical con drenaje percutáneo por servicio de radiología.

En la Figura 5 presentamos un esquema, del concepto de terapia asistida con vacío y estoma flotante.

\section{Discusión}

Una ostomía es un tratamiento que, si bien es altamente eficaz y en muchas ocasiones una necesidad que salva vidas, se comporta como una agresión física y psíquica que afecta la integridad corporal, la capacidad funcional y la calidad de vida, suponiendo un gran impacto en la vida diaria del paciente p $^{5-7}$.

Existen relatos anecdóticos del tratamiento de heridas y obstrucciones intestinales, tal como lo reporta Praxagorus en el 400 a.C., realizando una incisión sobre el vientre y el intestino para el tratamiento de la obstrucción intestinal y permitir el drenaje de la materia fecal retenida. Hipócrates (primo de Praxagorus), abogó por un supositorio de miel ungido con la hiel de un toro y seguido de un enema, si este método fallaba, la insuflación rectal, con un fuelle de un herrero era prescrito. Susruta en el 600 a. C. trataba estas heridas mediante la aplicación de las tenazas de la hormiga negra, aproximando la solución de continuidad, lavando el intestino lesionado $\mathrm{y}$, posteriormente, reintroduciéndolo en la cavidad abdominal. Dolaeus recomendaba beber una mezcla con el estiércol de caballo "porque el excremento expele excremento", también recomendaba frotar el abdomen con piel de una serpiente hervida en aceite o vino. Otros expertos indicaban la insuflación anal de humo de tabaco en el tratamiento de la obstrucción intestinal, Ambrosio Paré trataba la obstrucción intestinal con grandes dosis de mercurio crudo por

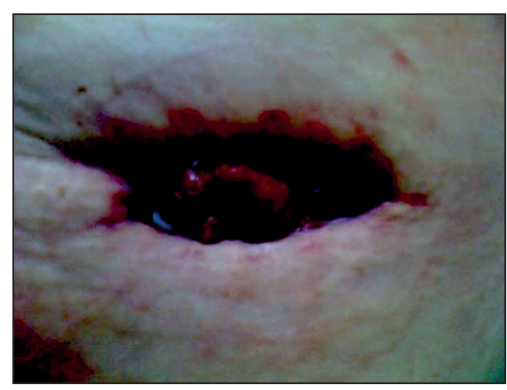

Figura 4. Resolución de absceso periostomal y cierre de espacio muerto secundario.

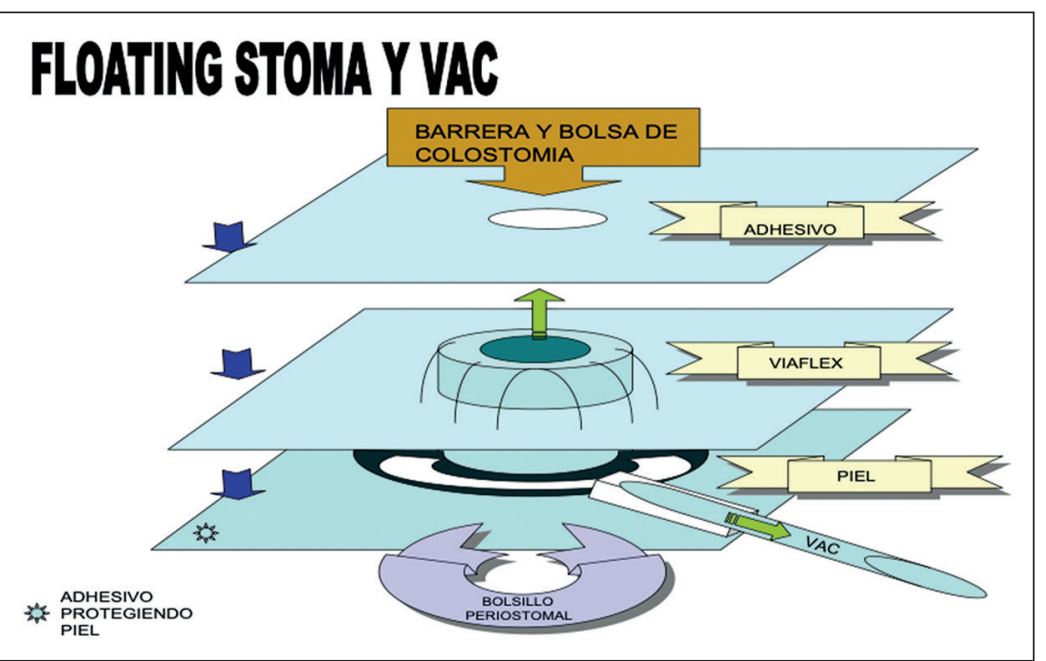

Figura 5. Esquema por planos del concepto a tratar.

vía oral, con la creencia de que el peso de los metales pesados corregía la obstrucción ${ }^{8-10}$.

En 1757, Lorenz Heister recomendó la exteriorización del intestino traumatizado. La primera ostomía fue realizada por Duret en 1793; Benjamin Well creó una ostomía de doble cañón para prevenir la estenosis del estoma. Penell (1850) construyó una sigmoidostomía para tratar una fístula recto-vesical atribuible a enfermedad inflamatoria intestinal (EII), mientras que Mayo-Robson (1893) practicó una colostomía inguinal en una colitis ulcerativa (CU), cuando la apendicostomía o la cecostomía se indicaban para la irrigación terapéutica del colon ${ }^{2,10}$.

En 1889 la primera ileostomía previa a una resección cólica estableció el concepto del manejo para colitis ulcerosa, hecha por los hermanos Strauss. Posteriormente fue Campbell, quien, suturando la mucosa a la piel, inició la construcción de colostomías mucosas e ileostomías de eversión, desarrolladas simultáneamente en Londres y Cleveland ${ }^{2}$.

Existen cerca de 1,5 millones de pacientes con ostomías intestinales en los Estados Unidos y 
100.000 nuevas son creadas anualmente. De estas, el $51 \%$ son colostomías, $35 \%$ ileostomías y $12 \%$ urostomías. Debido a los diferentes productos de estas, como son los fluidos intestinales con enzimas y contenidos alcalinos asociados a flora bacteriana intestinal, cambia drásticamente la piel y el tejido subcutáneo, causando desde lesiones por contacto en piel, sobreinfección, hasta colecciones en planos profundos ${ }^{11,12}$.

La celulitis paraostomal de origen bacteriano es rara. El agente causal en su mayoría es el Staphylococos aureus. El tratamiento con antibióticos sistémicos va dirigido a organismos Gram positivos. Las flictenas paraostomales, el dolor y el eritema ocurren secundario a la ruptura espontánea de la unión mucocutáneas. La flora intestinal es la principal causa de absceso, secundario a la presencia de una fístula mucocutánea. Todos los estomas que se presentan con abscesos paraostomales deberían ser resecados hasta la unión mucocutánea, en lo posible para evitar el "pouching" que puede ser resultado de la aproximación cercana de la herida y el estoma. Dependiendo de la severidad del proceso, se pueden realizar estudios complementarios con ecografía o tomografía abdominal contrastada; estas pruebas pueden demostrar colecciones asociadas ignoradas, masas inflamatorias, y la fístula, ayudando en el diagnóstico y el tratamiento del paciente, como se puede observar en nuestro caso ${ }^{5,12}$.

La retracción de la ostomía se presenta en alrededor del 5\%, puede ocurrir en pacientes con obesidad, infecciones paraostomales crónicas, estenosis de la ostomía, factores que producen la desinserción de los puntos de fijación del ostoma o la sección completa del asa de la ostomía, como en el caso presentado. La prevención inicial se puede lograr usando anillos de protección cutánea alrededor de la ostomía ${ }^{13}$.

Cuando la lesión se encuentra instaurada el tratamiento consiste en la observación, si la retracción es parcial, esperando la coalescencia coloparietal. Cuando la retracción es mayor o si ha desaparecido el ostoma se debe reintervenir al paciente para reconstruir un nuevo ostoma. Deben tomarse los cuidados para reducir al mínimo la contaminación intraperitoneal, evitando la salida de contenido intestinal, limpiando y lavando el campo operatorio. En todos los casos la zona retraída y con afectación del estado de la pared será resecada, rehaciéndose la colostomía en forma terminal. Si la primera colostomía fue lateral su transformación en terminal y abocar el cabo distal en otro lugar resultará más seguro, lo cual se puede evitar aplicando nuestra técnica de flotaing stoma con terapia al vacío ${ }^{13,14-17}$.
Los abscesos paraostomales ocurren en el 10\% al $25 \%$ de los casos de complicaciones y se relacionan con la inoculación parietal o a la contaminación de un hematoma paraostomal. Está relacionada a una mala preparación del colon por lo que es más frecuente en operaciones de urgencia, por lo cual se recomienda mantener el asa de la colostomía cerrada y recién hacer la apertura al final de la operación. Cuando no es imperioso abrir la ostomía, el diferir su apertura $24 \mathrm{~h}$ permite la adhesión del intestino a la pared disminuyendo la posibilidad de contaminación. Todas estas medidas han emergido como forma de evitar estas complicaciones y forman parte de un tratamiento preventivo ${ }^{15,17}$.

Las colecciones paraostomales que producen cavidades de espacio muerto al ser drenadas, pueden ser tratadas aplicando una solución de Milton (hipoclorito de sodio al 1\%) para esterilizarlas, sin embargo, esto puede ser doloroso y traumático para el paciente, mientras que con el floating stoma más la terapia al vacío, se logra retirar de forma continua los detritus y bacterias de estas colecciones y a su vez generar tejido de granulación ${ }^{17,18}$.

El manejo de las complicaciones paraostomales en los últimos 200 años han sido los mismos conceptos, de control infeccioso mediante antibióticos desde que los hay, las múltiples curaciones a repetición hasta la formación lenta de tejido de granulación o la necesidad de reintervención quirúrgica cuando estas fallan; exponiendo al paciente a un riesgo quirúrgico. En los últimos años el avance en radiología nos ha permitido el drenaje percutáneo de las colecciones, con una solución parcial al problema $^{18}$.

El estudio más grande de floating stoma reportado recientemente en la literatura es el de Manterola et al., quienes mostraron una serie de 46 pacientes que requirieron procedimientos con una segunda revisión para verificar la vitalidad gastrointestinal después de la laparotomía de control de daños realizada para sepsis abdominal mayor, traumatismo abdominal e isquemia mesentérica en Chile entre el 2005 y $2014^{19}$. El estoma que a menudo se requiere en estas situaciones de emergencia es a veces bastante difícil de modelar debido a la retracción mesentérica, la inflamación abdominal mayor y/o la distensión intestinal, similar a lo ocurrido en los casos de complicaciones paraostomales como el nuestro ${ }^{19,20}$.

Varios estudios han reportado casos de cirugía abdominal no traumática en los que el tratamiento con presión negativa ha demostrado ser beneficiosos para el monitoreo cuantitativo y cualitativo de las secreciones abdominales, la prevención de la for- 
mación de abscesos intraabdominales, la reducción del entorno inflamatorio mediante la externalización de los exudados intraperitoneales y al mismo tiempo facilitando el cierre definitivo de la pared abdominal mediante la aplicación de tracción continua en la aponeurosis; principios que se aprovechan en nuestro caso con el fin de manejar el floating stoma y el absceso paraostomal.

Otros autores ya han informado sobre la posibilidad de utilizar la terapia de presión negativa con el 'estoma flotante' a nivel abdominal, sin embargo, en nuestro conocimiento este es el primer caso reportado en la literatura del uso del floating stoma combinado con terapia al vacío para el manejo de un estoma complicado con abscesos para ostomales. En el estudio de Manterola et al., reportan varios casos de cirugía de control de daños en los que los estomas, se modelaron mediante terapia al vacío, sin evidenciar ningún efecto perjudicial de la presión negativa en los segmentos del intestino externo (en particular sin isquemia), y no encontraron ningún problema técnico en particular ${ }^{19}$. Además, los autores descubrieron que los estomas se podían externalizar fácilmente a través de un orificio en la espuma de presión negativa, lo que facilita la conexión de la bolsa de recolección, ya que la espuma se hizo rígida por el vacío, tal como en nuestro paciente ${ }^{20}$.

Autores reportan una técnica utilizando una bolsa flotante de "Borraez". Esta técnica quirúrgica acorta el tiempo operatorio (un elemento importante para los procedimientos de control de daños), preserva la pared abdominal y permite el tiempo suficiente antes del segundo examen para determinar la necesidad de reparación intestinal o derivación con un estoma convencional, lo cual se aplica para nuestro caso como un control de daños para la sepsis de origen periostomal. La bolsa de polietileno de Borraez se sutura directamente a la piel aproximadamente 2 $\mathrm{cm}$ alrededor de la incisión de laparotomía o de la ostomía y debajo de esta se coloca el dispositivo de terapia al vacío. Esto acorta el tiempo operatorio, facilita la segunda intervención, preserva la aponeurosis y evita el riesgo de contaminación intra abdominal por fuga de la ostomía, como en nuestro paciente ${ }^{19,20}$.

Hay poca evidencia sobre el uso del floating stoma, sumado a la escasa evidencia sobre otras opciones para controlar temporalmente una fístula intestinal, especialmente en aquellas situaciones en las que no es posible exteriorizar el asa intestinal. Dentro de estas opciones encontramos simplemente dejar el intestino grapado in situ hasta el cierre definitivo, la 'fístula VAC', 'tubo VAC', nipple VAC $\mathrm{y}$ el método de silo, que constituye una especie de

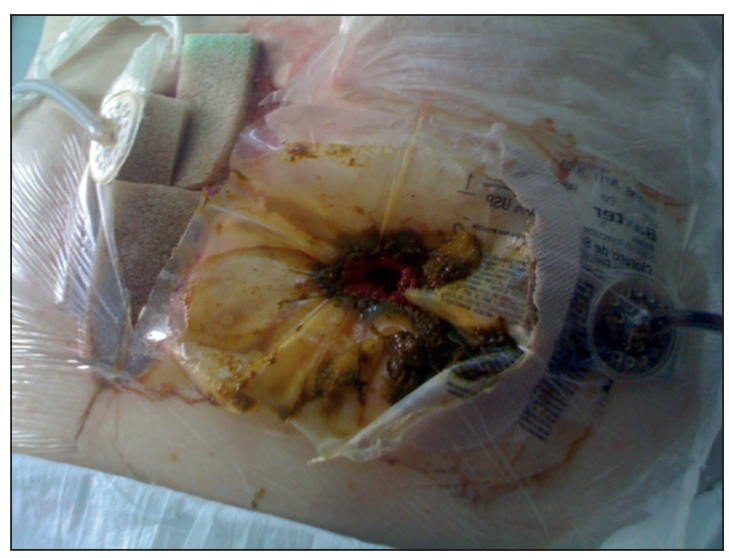

Figura 6. Aislamiento de cavidades por planos, no contaminación de materia fecal en estas. Estoma flotante en posición. “enterostomía con catéter”. Por esta razón, no es fácil discutir los resultados de nuestra experiencia con este caso, ya que como lo mencionamos anteriormente es el primero reportado para usando esta técnica con terapia al vacío para el manejo de una ostomía complicada ${ }^{19,20}$.

Con esto presentamos la aplicación de un concepto en manejo de la pared abdominal, como lo ha sido la bolsa de Borraez y el control de heridas por medio del sistema de terapia al vacío (Figura 6). Aplicándolos para un manejo adecuado y rápido de un proceso que llevaría semanas en curaciones diarias y que en múltiples ocasiones terminan en reintervenciones o frustración del equipo médico por resolución parcial del problema.

Mediante el "floating stoma" combinado con la terapia al vacío, se pueden resolver los abscesos periostomales totalmente, corregir la retracción y la pérdida de espacio muerto (bolsillos periostomales), formando tejido nuevo de granulación en $360^{\circ}$, mediante el principio de la terapia asistida por vacío y la solución con una neopared para el manejo de las heces de forma adecuada. Esto mediante un proceso no mórbido, práctico, ingenioso para resolver un problema en menor tiempo y de raíz.

\section{Conclusión}

El floating stoma es un recurso reservado para casos quirúrgicos especiales, como en los abscesos paraostomales que generan pérdida completa de la fijación de un estoma, deben ser realizados por equipos quirúrgicos con experiencia en el manejo del abdomen abierto con terapia de presión negativa. Se requieren estudios adicionales para confirmar su utilidad en este grupo específico de patologías. 


\section{Responsabilidades éticas}

Protección de personas y animales. Los autores declaran que para esta investigación no se han realizado experimentos en seres humanos ni en animales.
Confidencialidad de los datos. Los autores declaran que en este artículo no aparecen datos de pacientes.

Conflictos de interés: no hay.

\section{Bibliografía}

1. Subramaniam $\mathrm{MH}$, Liscum $\mathrm{KR}$, Hirshberg A. The Floating Stoma: A New Technique for Controlling Exposed Fistulae in Abdominal Trauma. J Trauma 2002;53:386-8.

2. Goverman J, Yelon JA, Platz JJ, Singson RC, Turcinovic M. The "Fistula VAC", a technique for management of enterocutaneous fistulae arising within the open abdomen: report of 5 cases. J Trauma 2006;60:428-31.

3. Velázquez-Gutiérrez J, Graffe C, Pausin A, Vargas M. VAC artesanal. Una técnica para el manejo de fístulas complejas con abdomen abierto. reporte de 2 casos. Rev Venez Cir. 2008;61:33-9.

4. Espinoza C, Pereira N, Benavides G, Rostion C. Ostomías abdominales en pediatría: una revisión de la literatura. Rev Ped Elec. 2008;5:38-48. ISSN 07180918

5. Núñez M, Marroquín R, Durán R, Lua T, Hermosillo J, Muciño I, et al. Complicaciones de las enterostomías: Revisión de 267 estomas. Rev Cir General 2001;23:148-53

6. Hierro J, Abed G, Galindo F. Complicaciones de las colostomías. Cirugía digestiva, F. Galindo 2009; III311:1-12.

7. Díaz JJ, Dutton WD, Ott MM, Cullinane DC, Alouidor R, Armen SB, et al. Eastern Association for the Surgery of Trauma: a review of the management of the open abdomen-part 2 "Management of the open abdomen". J Trauma 2011;71:502-12. doi: 10.1097/TA.0b013e318227220c.

8. Dubose JJ, Lundy JB. Enterocutaneous fistulas in the setting of trauma and critical illness. Clin Colon Rectal Surg. 2010;23:182-9. doi: 10.1055/s-00301262986.

9. Fabian TC. Damage control in trauma: laparotomy wound management acute to chronic. Surg Clin North Am. 2007;87:7393, vi.

10. Vatn MH, Sandvik AK. Inflammatory bowel disease. Scand J Gastroenterol. 2015;50:748-62.

11. Layton B, Dubose J, Nichols S, Connaughton J, Jones T, Pratt J. Pacifying the open abdomen with concomitant intestinal fistula: a novel approach. Am J Surg. 2010;199:e48-50. doi: 10.1016/j. amjsurg.2009.06.028.

12. Moesgaard FA. Vacuum-assisted closure of the open abdomen. Ugeskr Lager. 2007;169:1989.

13. Piazza RC, Armstrong SD, Vanderkolk W, Eriksson EA, Ringler SL. A modified "fistula-VAC" technique: management of multiple enterocutaneous fistulas in the open abdomen. Plast Reconstr Surg. 2009;124:453e-5e. doi: 10.1097/ PRS.0b013e3181bcf6d4.

14. Polk TM, Schwab CW. Metabolic and nutritional support of the enterocutaneous fistula patient: a three-phase approach.
World J Surg. 2012;36:524-33. doi: 10.1007/s00268-011-1315-0

15. Sauter ER. Temporary closure of open abdominal wounds by the modified sandwich-vacuum pack technique $(\mathrm{Br}$ J Surg. 2003;90:718-22). Br J Surg. 2003;90:1021-2. doi: 10.1002/bjs.4359

16. Schecter WP, Ivatury RR, Rotondo MF, Hirshberg A. Open abdomen after trauma and abdominal sepsis: a strategy for management. J Am Coll Surg. 2006;203:390-6. doi: 10.1016/j. jamcollsurg.2006.06.001

17. van $A s A B$, Navsaria $P$, Numanoglu A, McCulloch M. Modified sandwich vacuum pack technique for temporary closure of abdominal wounds: an african perspective. Acta Clin Belg. 2007;62 Suppl 1:215-9. doi: 10.1179/acb.2007.62. s1.029

18. Williams JW, Hiro SP. Management of gastroenteric anastomotic disruption. Am Surg. 1976;42:817-20

19. Manterola C, Flores P, Otzen T. Floating stoma: An alternative strategy in the context of damage control surgery. J Visc Surg. 2016;153:419-24. doi: 10.1016/j. jviscsurg.2016.06.003

20. Malgras B, Pasquier P, Savard DJ, Rouquie D, Baton $\mathrm{O}$, Boulanger T, et al. Intake of temporary abdominal closure with abdominal negative-pressure therapy in management of nontraumatic abdominal surgical emergencies to avoid stoma. Am Surg. 2014;80:E314-E316. 